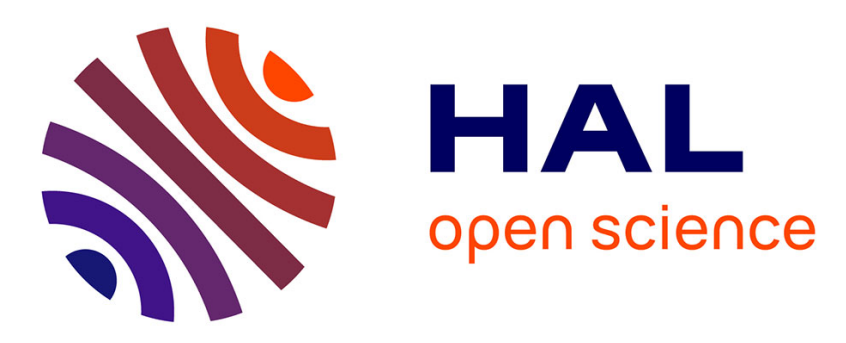

\title{
MusIC method enhancement by a sensitivity study of its performance: application to a lower limbs musculoskeletal model
}

\author{
Antoine Muller, Charles Pontonnier, Georges Dumont
}

\section{- To cite this version:}

Antoine Muller, Charles Pontonnier, Georges Dumont. MusIC method enhancement by a sensitivity study of its performance: application to a lower limbs musculoskeletal model. Computer Methods in Biomechanics and Biomedical Engineering, 2018, pp.1-13. hal-01904443

HAL Id: hal-01904443

https://hal.inria.fr/hal-01904443

Submitted on 24 Oct 2018

HAL is a multi-disciplinary open access archive for the deposit and dissemination of scientific research documents, whether they are published or not. The documents may come from teaching and research institutions in France or abroad, or from public or private research centers.
L'archive ouverte pluridisciplinaire HAL, est destinée au dépôt et à la diffusion de documents scientifiques de niveau recherche, publiés ou non, émanant des établissements d'enseignement et de recherche français ou étrangers, des laboratoires publics ou privés. 


\title{
MusIC method enhancement by a sensitivity study of its performance: application to a lower limbs musculoskeletal model
}

\author{
A. Muller ${ }^{\mathrm{a}}$, C. Pontonnier ${ }^{\mathrm{a}, \mathrm{b}}$ and G. Dumont ${ }^{\mathrm{a}}$ \\ ${ }^{a}$ Univ Rennes, CNRS, Inria, IRISA - UMR 6074, F-35000 Rennes, France; b́́coles de \\ Saint-Cyr Coëtquidan, 56380 Guer, France
}

\section{ARTICLE HISTORY}

Compiled October 24, 2018

\begin{abstract}
Providing a biomechanical feedback during experimental sessions is a real outcome for rehabilitation, ergonomics or training applications. However, such applications imply a fast computation of the biomechanical quantities to be observed. The MusIC method has been designed to solve quickly the muscle forces estimation problem, thanks to a database interpolation. The current paper aims at enhancing its performance. Without generating any database, the method allows to identify optimal densities (number of samples contained in the database) with respect to the method accuracy and the off-line computation time needed to generate the database. On a lower limbs model (12 degrees of freedom, 82 muscles), thanks to this work, the MusIC method exhibits an accuracy error of $0.1 \%$ with an off-line computation time lower than 10 minutes. The on-line computation frequency (number of samples computed per second) is about $58 \mathrm{~Hz}$. Thanks to these improvements, the MusIC method can be used to produce a feedback during an experimentation with a wide variety of musculoskeletal models or cost functions (used to share forces into muscles). The interaction between the subject, the experimenter (e.g. trainer, ergonomist or clinician) and the biomechanical data (e.g. muscle forces) in experimental sessions is a promising way to enhance rehabilitation, training or design techniques.
\end{abstract}

\section{KEYWORDS}

Musculoskeletal analysis; Motion; Computation time; Database; Biomechanical feedback; Interpolation.

\section{Introduction}

Providing a feedback based on biomechanical quantities during experimental sessions is a real outcome for rehabilitation, ergonomics or training applications. The interaction between the subject, the experimenter (e.g. trainer, ergonomist or clinician) and the biomechanical data (e.g. muscle forces) in experimental sessions is a promising way to adapt and enhance the subject performance according to the results of the analysis. However, such applications imply a fast computation of the biomechanical quantities to be observed - particularly muscle forces. The main challenge in estimating muscle forces is to treat the muscular redundancy question that leads to an underdetermined problem. This problem is classically solved by gradient-based numerical optimization schemes. In such an optimization scheme, the minimized cost function

CONTACT A. Muller. Email: antoine.muller@irisa.fr 
aims at mimicking the central nervous system behavior (An et al. 1984; Crowninshield and Brand 1981; Rasmussen et al. 2001) and the constraints represent the dynamical equilibrium of the musculoskeletal model and the maximal forces that the muscles can develop (Challis and Kerwin 1993; Challis 1997; Erdemir et al. 2007). Numerical optimization under non-linear constraints is usually computationally expensive. Thus, in most cases, the analysis is computed off-line thanks to pre-recorded and pre-processed motion data, that is unadapted to provide a feedback during the experimental session.

Several studies proposed solutions to provide a real-time visualization of muscle forces generating a given gesture. (Murai et al. 2010) proposed an adapted optimization scheme using electromyography (EMG) data and gathering muscles defined in the musculoskeletal model into functional groups in order to reduce the number of unknowns to optimize. This simulation required the use of EMG-sensors and made an important assumption by computing a unique activation level for all the muscles of a given functional group. As an alternative, (Van den Bogert et al. 2013) proposed to use a neural network trained to mimic quadratic optimization. The computation time was limited to ensure real-time computation. Both of these works proposed real-time musculoskeletal forces estimation thanks to the methods and assumptions described above, that limited the accuracy of their results.

To limit the computation time, (Pontonnier and Dumont 2009, 2010) proposed to interpolate pre-computed solutions from a muscle forces database. Limitations remained numerous since the method was applied to a single joint with only 4 muscles and a large work had to be done to extend the method to musculoskeletal models with more joints, muscles and muscular coupling - i.e. multi-articular muscles. Based on the same idea, (Muller et al. 2018) proposed the MusIC (Muscle forces Interpolation and Correction) method as a global solution to the muscle forces estimation problem. The results were very encouraging since the on-line computation frequency (number of samples computed per second) was multiplied by 10 with a mean error of $4 \%$ with regard to the optimization scheme. Three different cost functions (e.g. min-max, quadratic, cubic) were tested without any restriction.

Several issues needed to be solved to make the MusIC method being a credible solution to be used in a feedback setup. First, the method was applied on a 2 degrees of freedom (DOF) planar representation of the human arm (shoulder and elbow flexion) actuated by 12 muscles. This model was useful to evaluate the MusIC method performance for a large number of configurations but was not representative of a real musculoskeletal analysis, asking for more accurate and 3-dimensional models. Second, the off-line computation time - corresponding to the database generation time - was about 1 hour. Since one database has to be generated per subject, as each scaled model needs its own muscle force database in this method, the database generation has to be computed after the calibration of the model, that is in every case the first step of the experimental session. Therefore, 1 hour of waiting time per subject is not realistic for an effective use of the method in an experimental session with direct biomechanical feedback. Moreover, the database generation time grows with the complexity of the models to simulate due to the number of lines of action and degrees of freedom.

In order to circumvent these limitations, the current paper proposes to adapt and enhance the usability of the MusIC method for a complex 3-dimensional musculoskeletal model. First, the MusIC method has been applied to a lower limbs musculoskeletal model available in the AnyBody Modelling System (Damsgaard et al. 2006) to assess both its accuracy and computation time. Next, a sensitivity study has been conducted to find a trade-off between the MusIC method accuracy and the off-line computation time needed for the database generation. This method is able to find a priori - without 
generating databases - the databases exhibiting the best trade-offs between accuracy and computation time.

The article begins with a short presentation of the MusIC method. Second, assumptions are formulated to test if a database density can be decided without generating the associated database. Third, additional parameters are introduced to adjust the database structure in order to better control the database discretization. Then, the method is applied on a 3-dimensional lower limbs musculoskeletal model on a large set of database densities and evaluated by generating results of the MusIC method for all of these densities. Last, the results of accuracy and computation time are presented and discussed.

\section{Materials and methods}

In the following section, we consider a generic musculoskeletal model composed of $n_{b}$ bodies, articulated by $n_{j}$ joints and actuated by $n_{m}$ muscles. The $\left(n_{j} \times 1\right)$ vector $\boldsymbol{q}$ contains the joint coordinates, the $\left(n_{j} \times 1\right)$ vector $\boldsymbol{\Gamma}$ contains the joint torques and the $\left(n_{m} \times 1\right)$ vector $\boldsymbol{F}$ contains the muscle forces.

\subsection{The MusIC method}

From joint coordinates $\boldsymbol{q}$ and joint torques $\boldsymbol{\Gamma}$, the MusIC method aims at computing a muscle forces vector solution $\boldsymbol{F}$ on the basis of a precomputed database. The method is composed of two steps: the off-line database generation - only based on the musculoskeletal model - and the on-line muscle forces estimation - based on the database and on motion data. The database generation consists in storing solutions of the optimization problem for a set of joint configurations. From a current configuration, a first estimation is computed thanks to a linear interpolation in the database. Then, a constrained optimization corrects it to satisfy the dynamic equilibrium - respect of equations linking the joint torques and the muscle forces - and the physiological limits of the muscles - muscles can only pull with maximal forces. Readers are referred to (Muller et al. 2018) for more details about the MusIC method.

\subsection{Influence of database density}

The influence of the density of the database on the accuracy and the off-line computation time of the MusIC method is investigated in this part. For a given joint configuration, the MusIC method uses the closest configurations stored in the database in order to interpolate the related muscle forces. The more the joint configuration is discretized in the database, the more the result given by the interpolation is close to the optimization one. Moment arms are variables which influence the results of the optimization. So we can assume that investigating the error made on moment arms computation throughout the joint space discretization may explain most of the error provided by the interpolation method. To assess the database accuracy and computation time without generating them, we assumed the two following hypotheses to be discussed:

[H1] the accuracy of the MusIC method is directly correlated to the accuracy of the moment arms computation throughout the joint space discretization; 
[H2] the densities with highest trade-off between accuracy and off-line computation time for a scaled model are the same as whose defined on a generic model. We define the term generic model as a non-scaled model. The parameters of the scaled model are calibrated according to the studied subject.

To investigate these assumptions, we propose to use a generic musculoskeletal model and to evaluate the error made on moment arms computation during the joint space discretization. Therefore if hypotheses [H1] and [H2] are valid, this error should be correlated to the error made in the MusIC method with a scaled model. Thus, we propose to validate the assumptions by evaluating the accuracy of the muscle forces estimation with regard to the discretization of the database for a given set of motions and subjects.

\subsection{Database structure adjustments}

Let us consider a joint to be analyzed, called primary joint. The secondary joints of this primary joint are those which have an impact on the primary joint actuation, i.e., in our case, which have an influence on the moment arms values of the muscles actuating the primary joint. Figure 1 illustrates the primary and secondary joints concepts on a simple musculoskeletal model.

Primary joint: joint 1

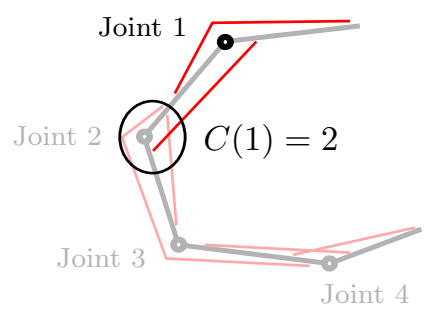

Primary joint: joint 3

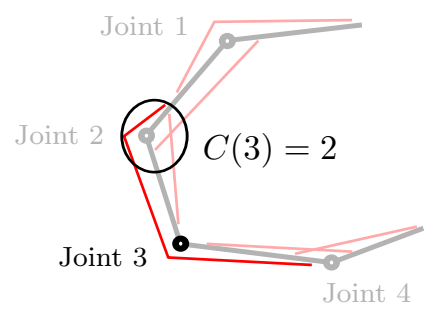

Primary joint: joint 2

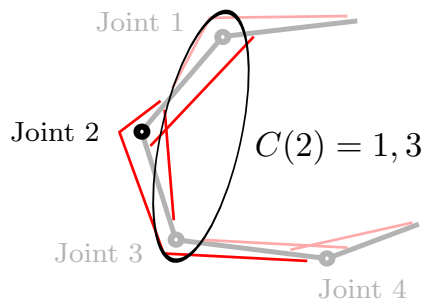

Primary joint: joint 4

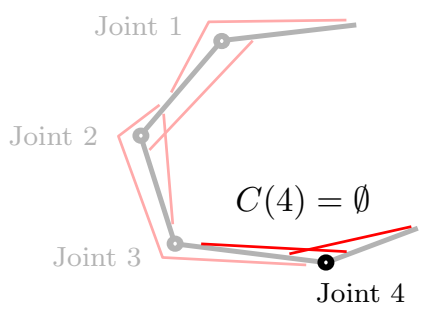

Figure 1. Secondary joints associated to each primary joint of a simple musculoskeletal model. For each primary joint, the secondary ones are those which have an influence on moment arms values of muscles actuating the primary joint. $C$ represents the index function of secondary joints. On this example, a bi-articular muscle between joint 1 and joint 2 induces that joint 2 is the secondary joint of joint 1 . The bi-articular muscles between joint 1 and joint 2 and between joint 2 and joint 3 induces that joints 1 and 3 are secondary joints of joint 2 . The bi-articular muscle between joint 2 and 3 induces that joint 2 is the secondary joint of joint 3 . Since all the muscles actuating joint 4 are mono-articular, no secondary joint is associated to this joint.

The precomputed database of the MusIC method contains a set of solutions of the optimization problem. These solutions are solved by considering each joint separately as primary joints, therefore one sub-database is generated per joint (left part Figure 2). In a sub-database, only the primary joint and its secondary ones are considered. The accessible, constrained by the joint limits, joint space associated to these joints is 
discretized to evaluate the evolution of the muscle forces estimation solution according to the joint configuration (central part Figure 2). For each of these joint configurations, the solution of the optimization problem is stored (right part Figure 2).

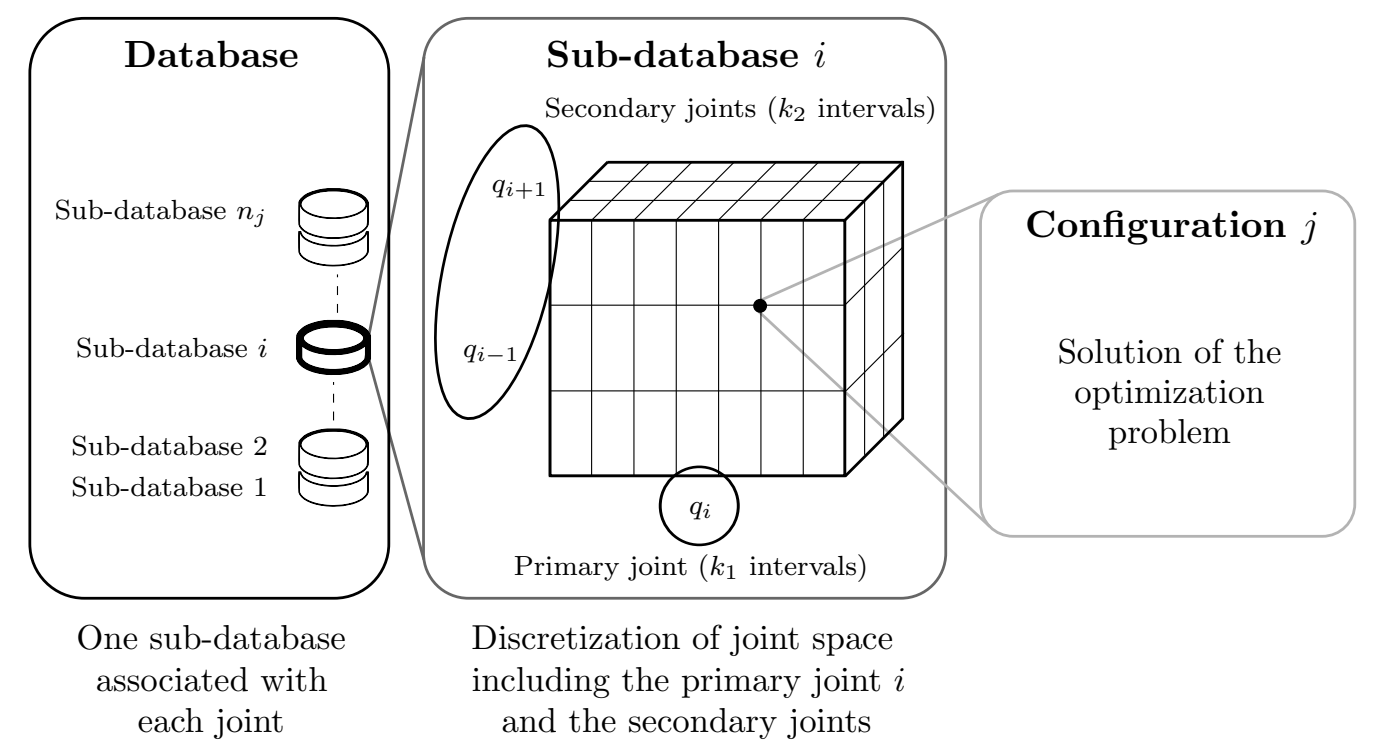

Figure 2. Databases structure composed of $n_{j}$ independent sub-databases (left part). Each of them is discretized at different joint configurations (central part) where the solution of the optimization problem is stored (right part).

We estimate the database density thanks to the number of samples that it contains. For each sub-database, we assume that the angular position of the primary joint has more impact on the moment arms values of this joint than the angular position of the secondary joints. As such, we propose to use different sampling density to discretize the primary joint amplitude and the secondary ones. The database density is defined thanks to the parameters $\left(k_{1}, k_{2}\right)$, where $k_{1}$ is the number of intervals for the discretization of the primary joint space and $k_{2}$ is the number of intervals for the discretization of the secondary ones. A regular sampling will be used in the following.

\subsection{Databases accuracy evaluation}

We evaluate the error made on moment arms computation in each database by interpolating moment arms for a large set of joint test configurations. Each joint coordinate is discretized into $k_{t}$ testing values between its physiological limits that leads to $k_{t}^{n_{j}}$ joint test configurations. For each joint database (defined by $k_{1}$ and $k_{2}$ values), moment arms are linearly interpolated from the joint configurations of the database for the $k_{t}^{n_{j}}$ joint test configurations. For the $\mathrm{j}$-th configuration, the interpolated value of the moment arms $R_{\text {interp }, j}$ is computed and compared to its reference value $R_{r e f, j}$. $R_{r e f, j}$ is computed thanks to its analytical expression, obtained from the analytical description of muscles anatomical references (origins, insertions and via points).

Finally, for a specified $\left(k_{1}, k_{2}\right)$ discretization, the error made on moment arms computation is evaluated by averaging the absolute difference between $R_{r e f}$ and $R_{\text {interp }}$ for all $k_{t}^{n_{j}}$ joint configurations (1). Thus, with regard to [H1], the lower this difference is, the higher the accuracy of the MusIC method should be. 


$$
\Delta R_{\text {dis }}=\frac{1}{k_{t}^{n_{j}}} \sum_{j=1}^{k_{t}^{n_{j}}}\left|R_{\text {ref }, j}-R_{\text {interp }, j}\right|
$$

$\Delta \tilde{R}_{d i s}$ is defined as the relative error of $\Delta R_{\text {dis }}$ with regard to the minimal value of all databases discretizations (2). $\Delta R_{d i s}^{\min }$ corresponds to the minimal value of $\Delta R_{\text {dis }}$. $\Delta \tilde{R}_{d i s}$ is called the moment arm error.

$$
\Delta \tilde{R}_{d i s}=\frac{\Delta R_{d i s}}{\Delta R_{d i s}^{\min }}-1
$$

Once the moment arms errors are evaluated, [H1] and [H2] are challenged through the application of experimental data to several databases generated with different densities. For each database, the accuracy of the MusIC method is associated with the off-line computation time (time used to generate the database).

As the MusIC method aims at emulating an optimization method, we evaluate its accuracy by computing, with the interpolated muscle forces solution, the value of the cost function $\epsilon_{c f}$ of the optimization method used to generate the solutions of the database. To facilitate the results exploitation, as done previously for the moment arms computation, $\epsilon_{c f}$ is defined as the relative error on $\epsilon_{c f}$ with regard to the minimal $\epsilon_{c f}$ value of all the databases of the study (3). $\epsilon_{c f}^{\min }$ corresponds to the minimal value of $\epsilon_{c f} \cdot \tilde{\epsilon_{c f}}$ is called the cost function error.

$$
\tilde{\epsilon_{c f}}=\frac{\epsilon_{c f}}{\epsilon_{c f}^{\min }}-1
$$

To validate the assumptions $[\mathbf{H 1}]$ and $[\mathbf{H 2}]$, a Spearman correlation coefficient between moment arm errors $\Delta \tilde{R}_{d i s}$ and cost function errors $\tilde{\epsilon_{c f}}$ for a given database discretization is computed.

Last, the database generation time is stored for each database generation. Indeed, the accuracy of the MusIC method is assumed to depend on the database density ([H1]). In a similar manner, since each sample computation in the database needs to solve an optimization problem, the database generation time - off-line computation time - grows with the number of samples to populate the database and would normally be correlated with the accuracy of the method.

\subsection{Application}

The method proposed above has been applied on a musculoskeletal model commonly used for analysis of lower limbs activities (Figure 3) - e.g. in the AnyBody Modelling System software (Damsgaard et al. 2006). It is composed of 82 muscles and actuates 6 joints containing 12 DOF - 3 for the hip, 1 for the knee and 2 for the ankle. This model uses a majority of poly-articular muscles and allows the method to be tested with a significant muscular coupling.

First, a generic version of this model close the 50th percentile - a size of $180 \mathrm{~cm}$ was used to assess the error realized on the interpolated moment arms. From this 
generic model, a set of 28 joint space discretizations was used. Each discretization corresponds to a couple of $\left(k_{1}, k_{2}\right)$ values - $(2,2),(3,2),(4,2),(5,2),(8,2),(10,2)$, $(15,2),(20,2),(30,2),(3,3),(4,3),(5,3),(8,3),(10,3),(15,3),(20,3),(30,3),(4,4)$, $(5,4),(8,4),(10,4),(15,4),(20,4),(5,5),(8,5),(10,5),(15,5)$ and $(20,5)$. For each discretization, the density was stored and the moment arm error was evaluated thanks to the equations (1) and (2). For this application, the parameter $k_{t}$ was set at the maximal value of $k_{1}$ and $k_{2}: k_{t}=30$. As the generic musculoskeletal model used is symmetric, the moment arm error is computed by only considering the muscles of one leg.

\begin{tabular}{c|c} 
& Number \\
\hline Muscles & 82 \\
\hline \hline Mono-articular & 8 \\
\hline Bi-articular & 16 \\
\hline Tri-articular & 40 \\
\hline Quadri-articular & 18
\end{tabular}

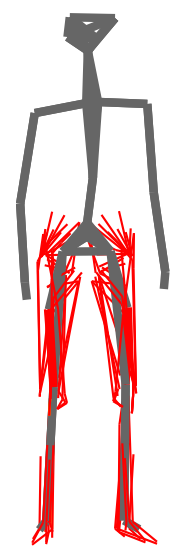

Figure 3. Representation of the musculoskeletal model used in this application. The model exhibits 12 DOF and 82 muscles.

Second, the hypotheses were challenged by applying the MusIC method to scaled models using experimental data. To this end, 10 male participants $(27.5 \pm 5$ years old, height: $180 \pm 7 \mathrm{~cm}$, mass: $74.1 \pm 7.3 \mathrm{~kg}$ ) participated to an experimentation. All subjects signed an informed consent form before participation and an anonymization protocol was followed for data safeguarding. Each subject was asked to mimic an avatar performing a standardized motion in a video (Figure 4). This video guide was designed to activate sequentially each degree of freedom. The motion was captured thanks to 16 Vicon ${ }^{\circledR}$ cameras $(100 \mathrm{~Hz})$ and 47 motion capture markers placed on standardized anatomical landmarks as recommended by the International Society of Biomechanics (ISB) (Wu et al. 2002, 2005). Moreover, the ground reaction forces were measured with two AMTI ${ }^{\circledR}$ force platforms $(1000 \mathrm{~Hz})$, each supporting one foot.

From experimental markers position, the geometrical parameters of the model were scaled thanks to an optimization-based calibration method (Muller et al. 2016). The inertial parameters of the model were deducted and scaled from an anthropometric rule (Dumas et al. 2007). Each scaled model corresponds to this calibrated model. The joint coordinates were computed using a global optimization method ( $\mathrm{Lu}$ and O'Connor 1999) and the joint torques were computed using a recursive Newton-Euler algorithm (Featherstone 2014) where measured ground reaction forces were considered as external forces.

For each subject, the 28 datab ses, corresponding to the $\left(k_{1}, k_{2}\right)$ couples described above, were generated only for validation. The corresponding time generation was stored for each database. The cost function used to generate the databases was a classical polynomial criterion corresponding to the cubic sum of the normalized muscle forces. Muscles forces were normalized by their maximum values based on anthropometric tables (no force-length nor force-velocity relationships were used here). Thanks 


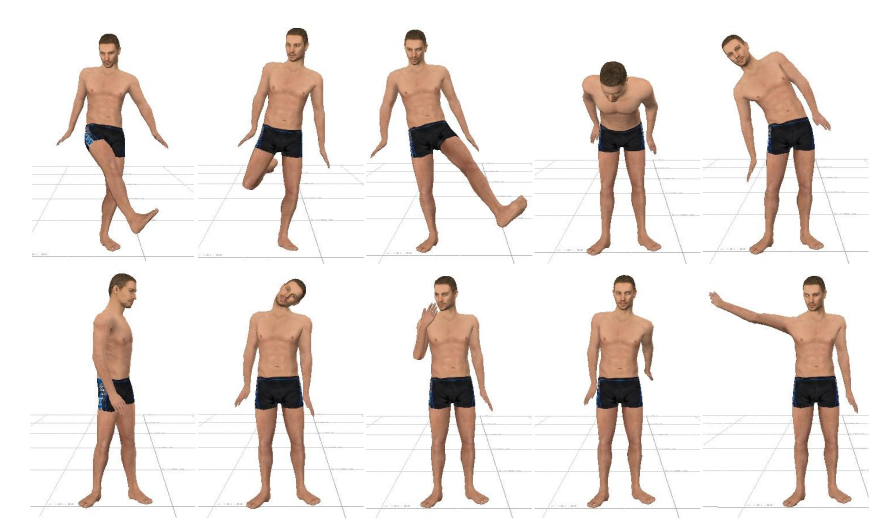

Figure 4. Different postures of the video guide. The video was generated using an avatar animated from motion capture data.

to the MusIC method (Muller et al. 2018), the muscle forces were estimated from each database previously described. The accuracy was then evaluated thanks to equation (3).

\section{Results and discussion}

\subsection{Optimal densities}

Considering the generic model, the moment arm error of a given database - evaluated by $\Delta \tilde{R}_{d i s}$ - has been plotted with regard to the number of samples of the associated database, directly computed from $k_{1}$ and $k_{2}$ (Figure 5). As expected, the moment arm error is directly linked to the database density. The higher the level of discretization is, the higher is the accuracy of the moment arms interpolation. Densities with $k_{2}=$ 2 belonging the Pareto frontier contain a small number of samples. Therefore, the corresponding off-line computation time is low. However, the errors made on moment arms computation can be more than ten times higher than the ones obtained the most accurate densities. In opposite, optimal densities with $k_{2}=5$ enable to obtain the highest accuracies. In return, with regard to the number of samples, the off-line computation time is higher. The optimal densities with $k_{2}=3$ or $k_{2}=4$ provide an interesting trade-off between the accuracy of the moment arms evaluation and the number of samples. Thus, a Pareto frontier appears in Figure 5.

Figure 6 depicts the MusIC method results obtained from the experimental data. First of all, the generation of the databases shows that the time generation is directly linked to the number of samples.

Then, considering figures 5 and 6 , the moment arm errors $\Delta \tilde{R}_{d i s}$ and cost function errors $\tilde{\epsilon_{c f}}$ exhibit similar trends with regard to the database discretization and computation time. The Spearman correlation between the mean of $\tilde{\epsilon}_{c f}$ and $\Delta \tilde{R}_{d i s}$ is $89 \%$ for each database. These results seem to confirm assumptions [H1] and [H2] previously described. Indeed, these strong correlations indicate that the MusIC method accuracy is correlated to the moment arms interpolation accuracy. Moreover, the MusIC method accuracy is barely affected by the model scaling since this correlation is computed between moment arms interpolated from a generic model and the MusIC method results applied to scaled models. In addition, the Spearman correlation between the standard deviation of $\tilde{\epsilon}_{c f}$ and $\Delta \tilde{R}_{d i s}$ is $79 \%$ for each database. Thus, more 


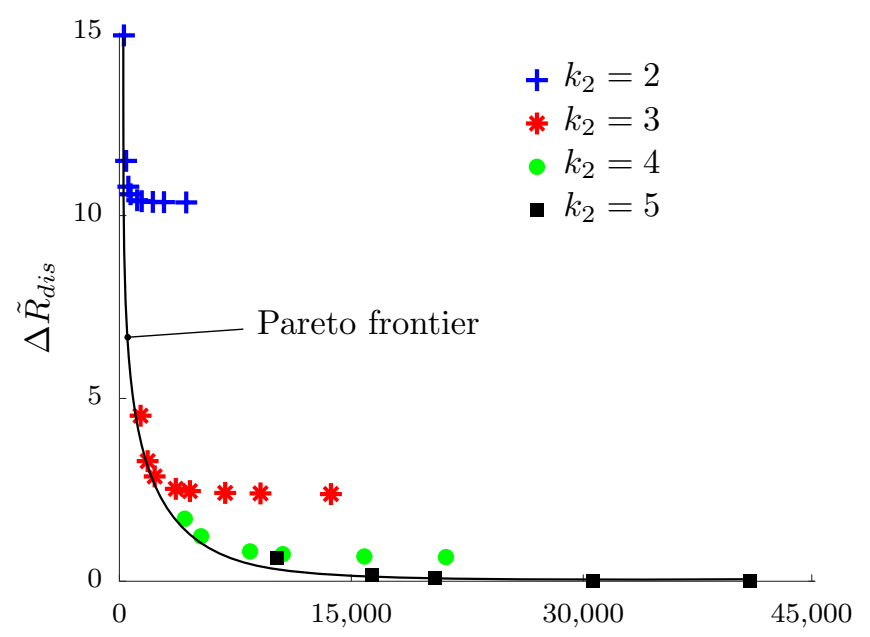

Number of samples of the discretization

Figure 5. Moment arm error $\Delta \tilde{R}_{d i s}$ for each joint space discretization. To facilitate the graph interpretation, we have chosen to associate one color for all $\left(k_{1}, k_{2}\right)$ parameters with the same $k_{2}$ value. Thus, the blues crosses correspond to the $(2,2),(3,2),(4,2),(5,2),(8,2),(10,2),(15,2),(20,2)$ and $(30,2)$ values; the red stars correspond to the $(3,3),(4,3),(5,3),(8,3),(10,3),(15,3),(20,3)$ and $(30,3)$ values; the green dots correspond to $(4,4),(5,4),(8,4),(10,4),(15,4)$ and $(20,4)$ values and the black squares correspond to $(5,5)$, $(8,5),(10,5),(15,5)$ and $(20,5)$ values. The number of samples of the discretization is directly related to $k_{1}$ and $k_{2}$.

the density is important, more the magnitude of the error is low. The validation of the two assumptions [H1] and [H2] indicates that optimal densities of the database can be directly deducted from the moment arm error. Since assumptions [H1] and [H2] are validated, the figure 5 can be used to find an optimal database density $\left(k_{1}, k_{2}\right)$ a priori, meaning there is no need to compute the database to have an realistic idea of its accuracy. Therefore, depending on the target application, one may choose a given density for a given level of performance (computation time, accuracy).

For example, for the model used in the current study, a cost function error of $0.1 \%$ could be obtained with a database generation time lower than 10 minutes by using the database with the $(4,4)$ density.

\subsection{Practical considerations}

The application presented in the current paper has been computed on a classical laptop (Intel Core i7 $2.8 \mathrm{GHz}$ processor) with Matlab ${ }^{\circledR}$. For this musculoskeletal model (exhibiting 82 muscles, 6 joints and $12 \mathrm{DOF}$ ), once the database generation is done, the on-line muscle forces computation frequency is about $58 \mathrm{~Hz}$ with the MusIC method and $1.6 \mathrm{~Hz}$ with a classical optimization method - for comparison purpose, the stopping criterion of the optimization method is selected to obtain the same level of accuracy as the MusIC method using the highest database density. Therefore, with a sufficiently low database generation time, the MusIC method may be considered as a feedback tool for users or experts in training or ergonomics applications since it can provide in a really fast time a reliable information about the muscle forces involved in the studied motion.

The use of the cost function error as a metric to test [H1] does not guarantee the same muscle forces repartition. However, as shown in (Muller et al. 2018), a good 


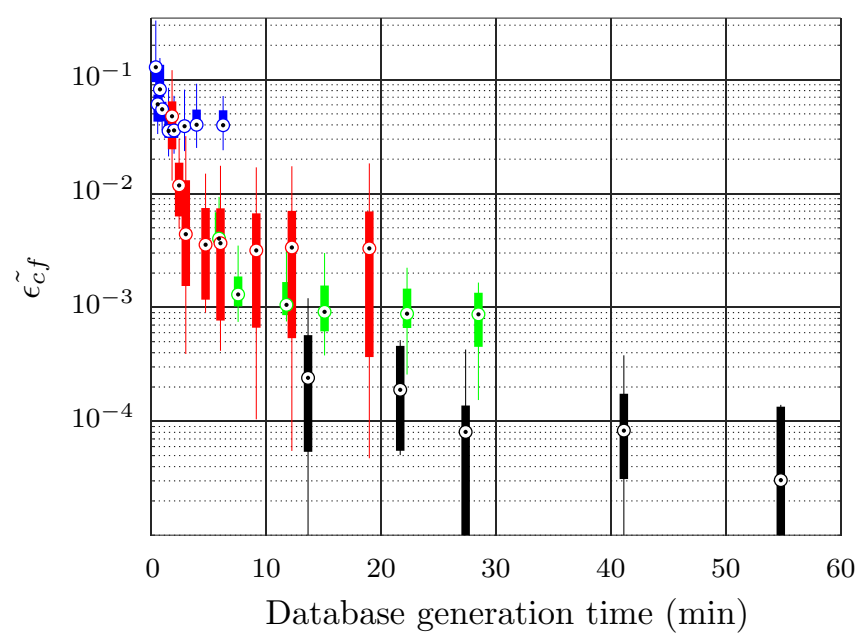

Figure 6. Application of the MusIC method to the experimental data. Cost function error $\epsilon_{c f}$ for each database discretization are plotted according to the corresponding database generation time. Let us notice that the vertical axis is plotted on a logarithmic scale. The blues box plots correspond to the $(2,2)$, $(3,2)$, $(4,2),(5,2),(8,2),(10,2),(15,2),(20,2)$ and $(30,2)$ values; the red ones correspond to the $(3,3),(4,3),(5,3)$, $(8,3),(10,3),(15,3),(20,3)$ and $(30,3)$ values; the green ones correspond to $(4,4),(5,4),(8,4),(10,4),(15,4)$ and $(20,4)$ values and the black ones correspond to $(5,5),(8,5),(10,5),(15,5)$ and $(20,5)$ values.

optimality of the cost function tends to obtain muscle forces close to those computed thanks to an optimization method.

When performing a musculoskeletal analysis session based on motion capture, the MusIC method can be used only if a database has been generated on the basis of the scaled model. Thus, this database generation has to be done after the scaling of the model. Since this scaling implies an experimental data collection (Muller et al. 2017), the decrease of the generation time allows the database to be generated on the fly during an experimental session, between the experimental calibration of the model and the capture of the motions to be analyzed. This computation time might be used by the experimenters to explain the experimentation protocol to the subject. In this context, the database with the density corresponding to $\left(k_{1}, k_{2}\right)=(4,4)$ seems to be an good trade-off. It allows to obtain a cost function error of about $0.1 \%$ with a database generation time lower than 10 minutes.

The influence of the database density on the on-line computation time - evaluated thanks to the computation frequency - has not been mentioned yet. In the current application, the average computation frequency for all the motions, subjects and databases is $58 \mathrm{~Hz}( \pm 3 \mathrm{~Hz})$, tested on the same laptop. Thus, with regard to this small standard deviation, we can consider that it does not influence the choices made on database densities.

\subsection{Limits and perspectives}

Optimal densities obtained in this study are specific to this musculoskeletal model. However, the method proposed in this paper allows to find the optimal densities without generating any database. For this, no experimental data is necessary. Thus, this study could be performed, a priori to any experiment, and included in the experimental design and in the model choice.

Moreover, for a given joint space discretization, the accuracy of the MusIC method 
applied to a scaled model is correlated to the accuracy of the moment arms interpolation applied to a generic model.

The sizes of the subjects range between $171 \mathrm{~cm}$ and $190 \mathrm{~cm}$. The differences between the scaled and the generic model are restricted, since the generic model was representing a subject of $180 \mathrm{~cm}$. The validity of the assumption [H2] has to be tested with other more distant further from the 50th percentile than these tested subjects. Moreover, the assumption is only confirmed for a geometrical optimization-based scaling and for an anthropometric inertial scaling. In order to enhance the results of the musculoskeletal study, other works suggest to scale the inertial parameters from motion and ground reaction forces (Ayusawa et al. 2011; Hansen et al. 2014; Jovic et al. 2016) and the muscular parameters from isokinetic ergometer measures (Garner and Pandy 2003; Van Campen et al. 2014; Heinen et al. 2015, 2016). The use of a complete scaling - geometrical, inertial and muscular parameters (Muller et al. 2017) - is therefore an interesting complementary step to validate assumption [H2], and also to increase the accuracy of the musculoskeletal analysis.

The muscle model used in this paper is simplified since the maximal available forces are considered as constants. Therefore the muscles forces estimation was only influenced by the joint moment arms. A force-length or force-velocity relationships could be used to model the muscle physiological behavior (Zajac 1989; Rengifo et al. 2010). The use of a force-length relationship does not change the MusIC method structure. However, the study here should be extended to take into account the influence of the database density on the muscles length computation. The use of a force-velocity relationship would modify the MusIC method since the joint configuration space and the joint velocity space would have to be discretized for each sub-database. Thus, the study here should be extended to the muscles velocity computation.

Last, an alternative database generation method might be applied to decrease the database computation time without affecting the accuracy of the MusIC method. Indeed the discretization of the joint space may be non regular in order to favor the database density around joint configurations impacting more critically the solution. For example, moment arm sign changes are critical and need a high density of the database whereas extreme joint positions seem less sensitive and databases may be less dense at these locations. Such non regular discretization may be generated a priori for a given generic model by analyzing the sensitivity of moment arms changes with regard to joint configuration changes. However, the current study showed that accurate results can be obtained from low database densities. This result may mitigate the impact of a non regular discretization for models of this complexity. Non regular discretization may only be relevant for much more complex models.

\section{Conclusion}

The estimation of muscle forces using a precomputed database demonstrated significant computational benefits (Muller et al. 2018) with a minimal loss of accuracy. The current paper aimed at addressing the limitations of the MusIC method, mostly concerning the off-line computation time, to be used as a feedback during a musculoskeletal analysis session. The study modulated the database density to find a tradeoff between the accuracy of the method and the off-line computation time (database generation time) and validated the method on a 3-dimensions model currently used in musculoskeletal analysis. The method was developed to find a priori - without generating databases - the best trade-offs to be applied for a given model and ex- 
perimental design. It consists in using a generic model to evaluate the error made on moment arms computation during the joint space discretization. On the lower limbs musculoskeletal model available in the AnyBody Modelling System (Damsgaard et al. 2006 ), the optimal database density allows to obtain an accuracy error of $0.1 \%$ with a corresponding off-line computation time lower than 10 minutes on a standard laptop. The on-line computation frequency is about $58 \mathrm{~Hz}$. Therefore, thanks to this complementary work, the MusIC method may be used to provide a musculoskeletal analysis feedback in a really short time during a mocap-based musculoskeletal analysis session. In conclusion, this kind of method could allow musculoskeletal indicators to be integrated to an exercise or work pattern for sports training or ergonomics applications respectively.

\section{References}

An K, Kwak B, Chao E, Morrey B. 1984. Determination of muscle and joint forces: a new technique to solve the indeterminate problem. Journal of biomechanical engineering. 106(4):364367.

Ayusawa K, Venture G, Nakamura Y. 2011. Real-time implementation of physically consistent identification of human body segments. In: Robotics and Automation (ICRA), 2011 IEEE International Conference on. IEEE. p. 6282-6287.

Challis J. 1997. Producing physiologically realistic individual muscle force estimations by imposing constraints when using optimization techniques. Medical Engineering and Physics. $19(3): 253-261$.

Challis J, Kerwin D. 1993. An analytical examination of muscle force estimations using optimization techniques. Proceedings of the Institution of Mechanical Engineers, Part H: Journal of Engineering in Medicine. 207(3):139-148.

Crowninshield R, Brand R. 1981. A physiologically based criterion of muscle force prediction in locomotion. Journal of Biomechanics. 14(11):793-801.

Damsgaard M, Rasmussen J, Christensen ST, Surma E, De Zee M. 2006. Analysis of musculoskeletal systems in the AnyBody Modeling System. Simulation Modelling Practice and Theory. 14(8):1100-1111.

Dumas R, Cheze L, Verriest JP. 2007. Adjustments to McConville et al. and Young et al. body segment inertial parameters. Journal of biomechanics. 40(3):543-553.

Erdemir A, McLean S, Herzog W, van den Bogert AJ. 2007. Model-based estimation of muscle forces exerted during movements. Clinical biomechanics. 22(2):131-154.

Featherstone R. 2014. Rigid body dynamics algorithms. Springer.

Garner BA, Pandy MG. 2003. Estimation of musculotendon properties in the human upper limb. Annals of biomedical engineering. 31(2):207-220.

Hansen C, Venture G, Rezzoug N, Gorce P, Isableu B. 2014. An individual and dynamic body segment inertial parameter validation method using ground reaction forces. Journal of biomechanics. 47(7):1577-1581.

Heinen F, Lund ME, Rasmussen J, de Zee M. 2016. Muscle-tendon unit scaling methods of hill-type musculoskeletal models: An overview. Proceedings of the Institution of Mechanical Engineers, Part H: Journal of Engineering in Medicine. 230(10):976-984.

Heinen F, Sørensen SN, King M, Lewis M, de Zee M, Rasmussen J. 2015. Prediction of muscletendon parameters based on isokinetic measurements. In: Annual Meeting of the Danish Society of Biomechanics. Danish Society of Biomechanics.

Jovic J, Escande A, Ayusawa K, Yoshida E, Kheddar A, Venture G. 2016. Humanoid and human inertia parameter identification using hierarchical optimization. IEEE Transactions on Robotics. 32(3):726-735.

Lu TW, O'Connor J. 1999. Bone position estimation from skin marker co-ordinates using global optimisation with joint constraints. Journal of biomechanics. 32(2):129-134. 
Muller A, Germain C, Pontonnier C, Dumont G. 2016. A simple method to calibrate kinematical invariants: application to overhead throwing. In: ISBS-Conference Proceedings Archive; vol. 33.

Muller A, Haering D, Pontonnier C, Dumont G. 2017. Non-invasive techniques for musculoskeletal model calibration. In: Congrès Français de Mécanique.

Muller A, Pontonnier C, Dumont G. 2018. The MusIC method: a fast and quasi-optimal solution to the muscle forces estimation problem. Computer Methods in Biomechanics and Biomedical Engineering. 21(2):149-160.

Murai A, Kurosaki K, Yamane K, Nakamura Y. 2010. Musculoskeletal-see-through mirror: Computational modeling and algorithm for whole-body muscle activity visualization in real time. Progress in biophysics and molecular biology. 103(2-3):310-317.

Pontonnier C, Dumont G. 2009. Inverse dynamics method using optimization techniques for the estimation of muscles forces involved in the elbow motion. International Journal on Interactive Design and Manufacturing. 3(4):227-236.

Pontonnier C, Dumont G. 2010. Interpolating Muscle Forces in an Inverse Dynamics Approach. Symposium on Computer Methods in Biomechanics and Biomedical Engineering (CMBBE).

Rasmussen J, Damsgaard M, Voigt M. 2001. Muscle recruitment by the min/max criterion A comparative numerical study. Journal of Biomechanics. 34(3):409-415.

Rengifo C, Aoustin Y, Plestan F, Chevallereau C. 2010. Distribution of forces between synergistics and antagonistics muscles using an optimization criterion depending on muscle contraction behavior. Journal of biomechanical engineering. 132(4):041009.

Van Campen A, Pipeleers G, De Groote F, Jonkers I, De Schutter J. 2014. A new method for estimating subject-specific muscle-tendon parameters of the knee joint actuators: a simulation study. International journal for numerical methods in biomedical engineering. 30(10):969987.

Van den Bogert AJ, Geijtenbeek T, Even-Zohar O, Steenbrink F, Hardin EC. 2013. A realtime system for biomechanical analysis of human movement and muscle function. Medical \& biological engineering \& computing. 51(10):1069-1077.

Wu G, Siegler S, Allard P, Kirtley C, Leardini A, Rosenbaum D, Whittle M, D'Lima DD, Cristofolini $\mathrm{L}$, Witte $\mathrm{H}$, et al. 2002. Isb recommendation on definitions of joint coordinate system of various joints for the reporting of human joint motion-part I: ankle, hip, and spine. Journal of biomechanics. 35(4):543-548.

Wu G, Van der Helm FC, Veeger HD, Makhsous M, Van Roy P, Anglin C, Nagels J, Karduna AR, McQuade K, Wang X, et al. 2005. Isb recommendation on definitions of joint coordinate systems of various joints for the reporting of human joint motion-part II: shoulder, elbow, wrist and hand. Journal of biomechanics. 38(5):981-992.

Zajac FE. 1989. Muscle and tendon: properties, models, scaling, and application to biomechanics and motor control. Critical reviews in biomedical engineering. 17(4):359-411. 